\title{
Hybrid Protein-Glycosaminoglycan Hydrogels Promote Chondrogenic Stem Cell Differentiation
}

\author{
Vladimíra Moulisová, ${ }^{\dagger}$ Sara Poveda-Reyes, ${ }^{\ddagger}$ Esther Sanmartín-Masiá, ${ }^{\ddagger}$ Luis Quintanilla-Sierra, ${ }^{\S} \|$ \\ Manuel Salmerón-Sánchez, ${ }^{*}+{ }^{\dagger}$ and Gloria Gallego Ferrer*,* ${ }^{*}$
}

${ }^{\dagger}$ Division of Biomedical Engineering, School of Engineering, University of Glasgow, Rankine Bld, Oakfield Avenue G12 8LT, Glasgow, U.K.

${ }^{\ddagger}$ Centre for Biomaterials and Tissue Engineering (CBIT), Universitat Politècnica de València, Camino de Vera s/n. 46022 Valencia, Spain

${ }^{\S}$ BIOFORGE Group, Centro de Investigación Científica y Desarrollo Tecnológico, Universidad de Valladolid, Campus Miguel Delibes 47011 Valladolid, Spain

"Biomedical Research Networking Center in Bioengineering, Biomaterials and Nanomedicine (CIBER-BBN), Instituto de Salud Carlos III, C/Monforte de Lemos 3-5, pabellón 11, planta 0, 28029 Madrid, Spain

\section{Supporting Information}

ABSTRACT: Gelatin-hyaluronic acid (Gel-HA) hybrid hydrogels have been proposed as matrices for tissue engineering because of their ability to mimic the architecture of the extracellular matrix. Our aim was to explore whether tyramine conjugates of $\mathrm{Gel}$ and $\mathrm{HA}$, producing injectable hydrogels, are able to induce a particular phenotype of encapsulated human mesenchymal stem cells without the need for growth factors. While pure Gel allowed good cell adhesion without remarkable differentiation and pure HA triggered chondrogenic differentiation without cell spreading, the hybrids, especially those rich in HA, promoted chondrogenic differentiation as well as cell proliferation and adhesion. Secretion of chondrogenic markers such as aggrecan, SOX-9, collagen type II, and glycosaminoglycans was observed, whereas osteogenic, myogenic, and adipogenic markers (RUNX2, sarcomeric myosin, and lipoproteinlipase, respectively) were not present after 2 weeks in the growth medium. The most promising matrix for chondrogenesis seems to be a mixture containing $70 \% \mathrm{HA}$ and $30 \%$ $\mathrm{Gel}$ as it is the material with the best mechanical properties from all compositions tested here, and at the same time, it provides an environment suitable for balanced cell adhesion and chondrogenic differentiation. Thus, it represents a system that has a high potential to be used as the injectable material for cartilage regeneration therapies.

\section{INTRODUCTION}

Many tissues in the human body are not able to properly repair themselves or can only repair small injuries, as in the case, for example, of skin, ${ }^{1}$ heart, ${ }^{2}$ and cartilage. ${ }^{3}$ Tissue engineering looks for solutions to these problems by using materials or scaffolds as supports for the formation of new tissue. Before transplantation, these scaffolds can either be seeded with differentiated or undifferentiated cells or be acellular if neighboring cells can migrate to the site of the implant inside the material. Other factors (e.g., growth factors) or stimuli (e.g., mechanical, electrical, or magnetic forces) can be applied to the scaffold-cell system to induce cell differentiation and promote tissue repair. ${ }^{4}$

In this study, we focus on material systems that recapitulate the properties of soft tissues (e.g., cartilage, muscle, etc.). The cells in these tissues are within a highly hydrated extracellular matrix (ECM), which contains glycoproteins (such as collagen, elastin, and fibronectin) and glycosaminoglycans [GAGs, such as hyaluronic acid (HA), chondroitin 6-sulfate, and keratan sulfate], with a composition and topology that is tissuespecific. $^{5}$ For this, we synthesized injectable hydrogels by combining different proportions of gelatin (Gel) and HA, which are able to enzymatically cross-link. Gel is obtained by denaturation of collagen and has accessible functional groups that can react with other molecules. It contains the RGD sequence, which allows integrin-mediated adhesion. ${ }^{6} \mathrm{HA}$ is characterized by its high hydrophilicity, good lubrication capacity due to its high water sorption and retention, good biocompatibility, and low cell and protein adhesive properties.?

Different options have been studied for the cross-linking or functionalization of $\mathrm{HA}$ and Gel hydrogels by forming covalent bonds. These can be classified into three groups: chemical, photochemical, or enzymatic cross-linking. ${ }^{8-11}$ Both chemical and photochemical cross-linking can produce inflammation and

Received: September 4, 2017

Accepted: October 13, 2017

Published: November 7, 2017 


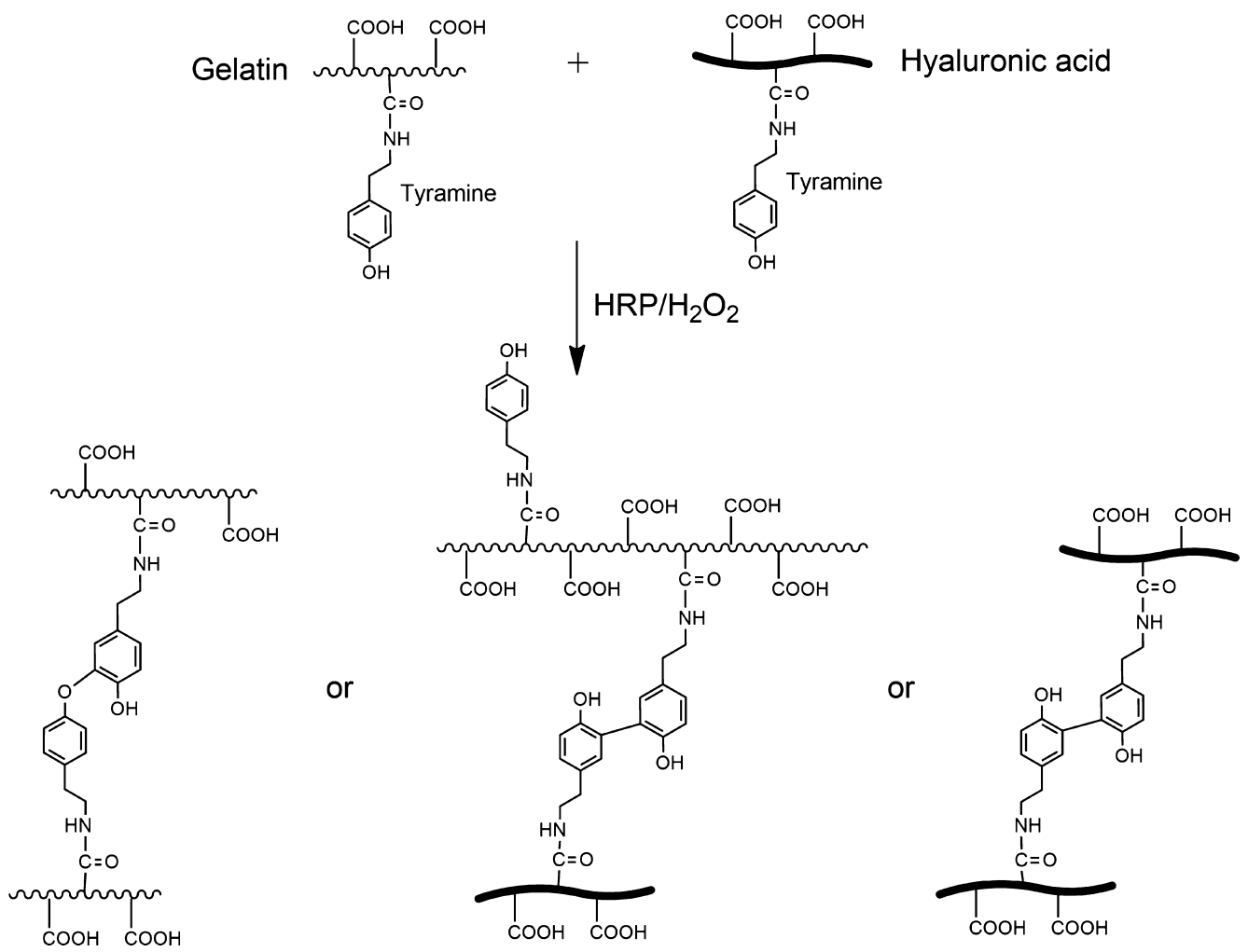

Figure 1. Enzymatic reaction of Gel-tyramine and HA-tyramine grafted composites by $\mathrm{HRP}$ and $\mathrm{H}_{2} \mathrm{O}_{2}$. When both the tyramine conjugates are mixed, random cross-linking reactions between Gel chains, HA chains, or Gel-HA chains are produced.

cell death, and their surgical procedure is more invasive than that required for enzymatic cross-linking, ${ }^{12,13}$ which allows in situ hydrogel formation; the precursor solutions can be injected directly into the defect, where the enzyme starts cross-linking without causing any cytotoxic effects. ${ }^{14}$

Previous studies combining Gel and HA have demonstrated their noncytotoxicity and potential for cell adhesion and spreading. ${ }^{7,15,16}$ Chen et al. embedded nucleus pulposus (NP) cells in chemically cross-linked Gel-HA hydrogels for 1 week, and their results showed good cell proliferation and cell synthesis of collagen type II, aggrecan, and Sry-type high mobility group box transcription factor 9 (SOX-9) (chondrogenic markers), biglycan and decorin (proteoglycans for ECM integrity), and hypoxia-inducing factor-1 (marker of NP cells). ${ }^{16}$ Camci-Unal et al. cultured human umbilical cord vein endothelial cells in methacrylated mixtures of Gel-HA, obtaining different cellular responses by changing the concentration of each component. ${ }^{17}$ These two references are examples of injectable materials (see also refs 18 and 19), which intend to mimic the composition of the natural ECM by mixing Gel and HA in different proportions. The most explored application of these systems has been in articular cartilage, likely due to the potential of HA to support chondrogenesis. ${ }^{20}$ However, none of these studies explored the application of these Gel-HA systems to trigger mesenchymal stem cell (MSC) differentiation. Rather, they revealed that encapsulated chondrocytes kept their phenotype within the gels but only in media containing chondrogenic growth factors, such as TGF$\beta 3 .{ }^{16,19}$ Other similar systems (collagen type II-HA or HA$\mathrm{Gel}$ ), which are based on noninjectable chemistries, have been used to investigate MSC differentiation in combination with chondrogenic growth factors. ${ }^{11,21}$ Only a few reports focused on the intrinsic chondrogenic potential of the matrices in the absence of growth factors, as was the case with the collagen type II-HA scaffolds reported by Murphy et al. ${ }^{22}$ or the cartilage decellularized ECM investigated by Burnsed et al. ${ }^{23}$ However, these are noninjectable preformed scaffolds obtained by lyophilization or solvent casting of aqueous solutions of macromolecules, followed by cross-linking. ${ }^{11,21,22}$ This initial drying not only generates some porosity but also triggers the organization of the macromolecules in a way that is very different from the one obtained in injectable hydrogels and which is kept after the addition of the cross-linker. Equilibrium water content (EWC), mechanical properties, and the way the cells interact with these hydrogels are very different from those of injectable hydrogels. ${ }^{24}$ Therefore, the results obtained on MSC differentiation in noninjectable hydrogels cannot be extrapolated to injectable hydrogels.

Encouraged by the positive results obtained in our previous work in enzymatically cross-linked Gel-HA hydrogels on myoblast differentiation, ${ }^{25}$ in this work, we evaluate the influence of hydrogel composition (proportion of Gel and $\mathrm{HA}$ ) on the differentiation of human MSCs (hMSCs) in the absence of growth factors. To the best of our knowledge, this is the first time that injectable Gel-HA hydrogels have been investigated as matrices for MSC differentiation without the addition of exogenous factors. The hydrogels are firstly physically characterized (rheology, swelling, and in vitro degradation), and their differentiation potential is then studied with encapsulated hMSCs. We assess whether the combination of both materials leads to an environment that promotes cell differentiation more efficiently than pure components in a growth medium (GM), that is, absence of specific complements such as growth factors. 

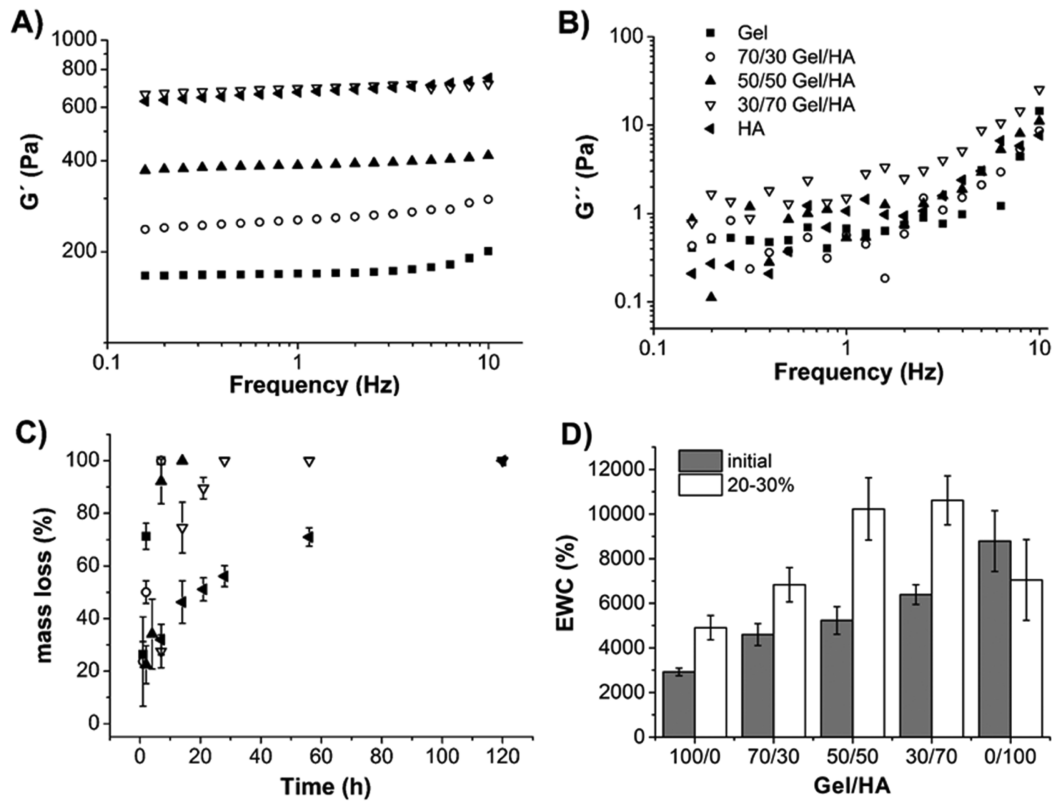

Figure 2. Rheological properties of Gel-HA hydrogels: (A) evolution of the storage $\left(G^{\prime}\right)$ and (B) loss moduli $\left(G^{\prime \prime}\right)$ as a function of the frequency of cross-linked hydrogels ( $1 \%$ strain). All measurements were carried out at $37^{\circ} \mathrm{C}$. Each curve corresponds to the average of three different samples. Enzymatic degradation study with $10 \mathrm{U} / \mathrm{mL}$ of hyaluronidase and $3 \mathrm{U} / \mathrm{mL}$ of collagenase: (C) percentage of mass lost with time for each Gel-HA hydrogel and (D) EWC of the Gel-HA hydrogels just after synthesis (initial) and after $20-30 \%$ of degradation of the matrix (20-30\%). Legend in (B) is also valid for $(\mathrm{A}, \mathrm{C})$.

\section{RESULTS}

Tyramine conjugates of Gel and HA form hydrogels by the covalent bond of the phenol groups of tyramine when horseradish peroxidase (HRP) and hydrogen peroxide $\left(\mathrm{H}_{2} \mathrm{O}_{2}\right)$ are added. ${ }^{27}$ When both the conjugates are mixed, hydrogels with different amounts of Gel and $\mathrm{HA}$ are obtained by the random reaction of Gel-HA, HA-HA, or Gel-Gel chains, as illustrated in Figure 1. Gelation times range from 2 to $4.5 \mathrm{~min}$, with an increase in the gelation time with the increase in the HA content. ${ }^{25}$ Gelation time in tyramine conjugates and other similar injectable hydrogels is usually not affected by the incorporation of cells, ${ }^{28}$ and we did not observe any significant change in the presence of cells. However, to ensure complete cross-linking of the hydrogels in the cell culture experiments, they were kept for 30 minutes at $37{ }^{\circ} \mathrm{C}$ before the addition of the culture medium. Successful tyramine grafting, tyramine substitution degree, and mean molecular weight of the tyramine conjugates of Gel and HA were determined by proton nuclear magnetic resonance spectroscopy, ultraviolet spectroscopy, and size exclusion chromatography, and the details can be found in our previous publications. ${ }^{25,29}$

2.1. Mechanical Properties. The shear modulus of the already cross-linked samples was measured. First, a strain sweep measurement was carried out to obtain the hydrogel linear viscoelastic range. ${ }^{30}$ No noticeable change was observed in $\left|G^{*}\right|$ with the strain amplitude throughout the whole amplitude strain range swept $(0.01-15 \%)$ in any of the gels (Figure S1). As can be seen, $\left|G^{*}\right|$ increases with the HA ratio, which has been reported previously in similar systems. ${ }^{18}$ As a trade-off between linearity and noise, subsequent dynamic frequency sweep tests were performed for $1 \%$ strain. The dependence of $G^{\prime}$ and $G^{\prime \prime}$ on the frequency for the hybrid gels has been plotted in Figures 2A and 2B, respectively. The storage modulus is the dominant contribution to $\left|G^{*}\right|$ because $G^{\prime} \gg G^{\prime \prime}$ in all gels, as has also been reported in thiolated Gel-HA hydrogels ${ }^{18}$ and in oxidized HA-Gel-adipic acid dihydrazide hydrogels. ${ }^{16}$ No significant dependence of $G^{\prime}$ on the frequency is observed for the experimental range of frequency swept. Again, the higher the ratio of $\mathrm{HA}$ in the hybrid gel the higher the storage modulus. No noticeable change was found in the loss modulus (with a value around $1 \mathrm{~Pa}$, regardless of the gel composition) until $2-3 \mathrm{~Hz}$, but it did increase at higher frequencies.

The value of $G^{\prime}$ at $1 \mathrm{~Hz}$ appeared in the range of several hundreds of $\mathrm{Pa}$ and increased with the percentage of HA from 172 to $789 \mathrm{~Pa}$ for pure Gel and HA, respectively (Table 1).

Table 1. Mechanical Properties of Hydrogels Determined by Rheometry $^{a}$

$\begin{array}{cccc}\text { Gel-HA hydrogel } & G^{\prime}(\mathrm{Pa}) & G^{\prime \prime}(\mathrm{Pa}) & \delta(\mathrm{deg}) \\ 100 / 0 & 172 \pm 38 & 0.9 \pm 0.3 & \sim 0.3 \\ 70 / 30 & 277 \pm 32 & 0.5 \pm 0.1 & \sim 0.1 \\ 50 / 50 & 366 \pm 28 & 0.5 \pm 0.1 & \sim 0.1 \\ 30 / 70 & 690 \pm 85 & 1.5 \pm 0.3 & \sim 0.1 \\ 0 / 100 & 789 \pm 220 & 1.0 \pm 0.1 & \sim 0.1\end{array}$

${ }^{a}$ Storage $\left(G^{\prime}\right)$ and loss $\left(G^{\prime \prime}\right)$ moduli and phase angle $(\delta)$ evaluated at a frequency of $1 \mathrm{~Hz}$, using $1 \%$ strain and at $37{ }^{\circ} \mathrm{C}$ for hybrids with different compositions.

Although the rheological properties of hydrogels were determined in the absence of cells, no changes in the shear storage moduli are expected when cells are incorporated. This was demonstrated by Kolesky et al. $^{31}$ using fibroblasts in methacrylated Gel and by Moshayedi et al. ${ }^{32}$ using neural progenitor cells in methacrylated HA. As far as the loss factor is concerned, an extremely low value (about $0.1^{\circ}$ ) was found for all hybrid gels, indicating a highly elastic energy storing capacity (Table 1).

2.2. Enzymatic Degradation of Gel-HA Hydrogels. The degradation of Gel-HA hydrogels was studied by enzymatic degradation with a $10 \mathrm{U} / \mathrm{mL}$ solution of 
hyaluronidase and $3 \mathrm{U} / \mathrm{mL}$ of collagenase in Dulbecco's phosphate-buffered saline (DPBS), these concentrations being within the range typically used before. ${ }^{11,17,33,34}$ A mixture of both the enzymes was used to reproduce a more relevant physiological environment than just using a single enzyme. Degradation kinetics was studied as the mass lost with time of immersion in the degradation solution (see Figure 2C). The Gel hydrogel degraded very rapidly; after $7 \mathrm{~h}$, there was no hydrogel left, whereas a longer time (5 days) was needed for HA. This trend has previously been reported by other authors. ${ }^{7}$ Gel-HA hybrids needed intermediate times to degrade. 70/30 and 50/50 Gel-HA hydrogels showed a degradation profile similar to the Gel hydrogel, and 30/70 fully degraded after $28 \mathrm{~h}$. These revealed an important role of Gel in accelerating degradation (even with only $30 \%$ of $\mathrm{Gel}$ in the gel) in comparison to pure HA. A reason for this could be that in hybrid gels, Gel is first degraded by collagenase, leaving spaces between some of the HA chains that improve the accessibility for hyaluronidase to degrade this component faster than in the bare HA hydrogel $(0 / 100)$.

To better understand how degradation influences the hydrogel structure, the EWC of different hydrogels at 20$30 \%$ degradation was compared with the EWC of hydrogels with no degradation (Figure 2D, Table 2). Initial hydrogel

Table 2. Initial EWC and Cross-Linking Density $\left(\rho_{x}\right)$ of the Hydrogels and Values after 20-30\% of Hydrogel

Degradation $\left(\mathrm{EWC}_{\mathrm{deg}}\right),\left(\rho_{x, \mathrm{deg}}\right)$

\begin{tabular}{ccccc}
$\begin{array}{c}\text { Gel-HA } \\
\text { hydrogel }\end{array}$ & EWC [\%] & $\mathrm{EWC}_{\mathrm{deg}}[\%]$ & $\begin{array}{c}\rho_{x} \\
{\left[\mathrm{~mol} / \mathrm{m}^{3}\right]}\end{array}$ & $\begin{array}{c}\rho_{x, \mathrm{deg}} \\
{\left[\mathrm{mol} / \mathrm{m}^{3}\right]}\end{array}$ \\
$100 / 0$ & $2924 \pm 169$ & $4908 \pm 542$ & 1.95 & 0.68 \\
$70 / 30$ & $4597 \pm 489$ & $6833 \pm 770$ & 1.14 & 0.54 \\
$50 / 50$ & $5232 \pm 620$ & $10228 \pm 1398$ & 1.11 & 0.33 \\
$30 / 70$ & $6390 \pm 439$ & $10615 \pm 1096$ & 0.93 & 0.38 \\
$0 / 100$ & $8790 \pm 1363$ & $7046 \pm 1812$ & 0.69 & 1.02 \\
\hline
\end{tabular}

swelling showed an increased water uptake with the higher HA content. This higher swelling is usually related to the lower cross-linking density of the polymeric network and chemical characteristics of the polymeric structure. ${ }^{6,7}$ EWC is increased in all compositions studied after degradation, except in the case of pure HA.

2.3. hMSCs Proliferation. Cells encapsulated within the hydrogels were viable during the time of the experiment, as demonstrated by the LIVE/DEAD assay on day 14 (Figure S2 of the Supporting Information). Cell morphology was monitored during the cell culture experiment on days 2, 7 , and 14 under the microscope. Figure 3 represents gels with embedded cells after 2 weeks in both culture media [GM and chondrogenic medium (CM)]; the details for the entire experiment $(2,7$, and 14 days) are shown in the Supporting Information (Figures S3.1 and S3.2). In the Gel hydrogel, the cells adhered and showed an elongated morphology in both culture media (GM and CM) from day 2 (Figures S3.1 and S3.2). In the case of $\mathrm{HA}$ and Gel-HA mixtures, the cells remained rounded on day 2 after seeding (Figures S3.1 and S3.2). In the GM, 70/30 and 50/50 hybrids started to show elongated and better attached cells on day 7 (Figure S3.1), whereas $30 / 70$ hydrogel facilitated cell-spreading later on as some elongated cells were observed on day 14 (Figures 3 and S3.1). In the CM, some cell elongation could be seen after 7 days of culture in Gel-HA hybrids (Figure S3.2). The pure HA

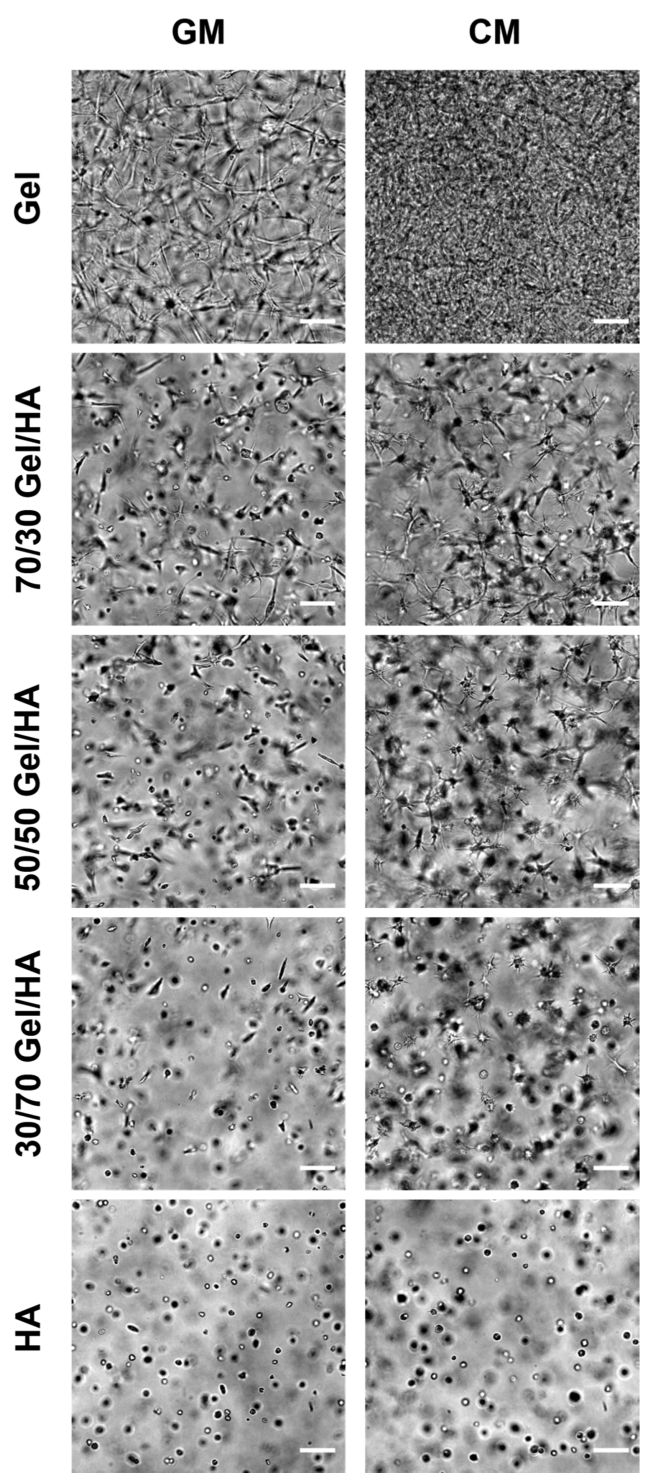

Figure 3. Phase contrast images of Gel-HA hydrogels with hMSCs cultured in GM and CM for 14 days. The scale bar is $100 \mu \mathrm{m}$.

hydrogel did not allow good cell attachment and spreading in any of the cell culture media, and the cells remained rounded during the entire experiment (Figures S3.1 and S3.2).

3-(4,5-Dimethylthiazol-2-yl)-5-(3-carboxymethoxyphenyl)-2(4-sulfophenyl)-2H-tetrazolium (MTS) assay (Figure 4) demonstrated that the cells did not proliferate in Gel in any of the culture media (GM and CM). However, a significant proliferation was measured for the hybrid gels in the GM (Figure 4A). In the case of pure HA, no proliferation was observed in the GM, and the number of cells diminished with the culture time, probably due to cell migration out of the hydrogel because of poor cell adhesion (Figure 4A). The presence of proliferating cells in the Gel-HA mixtures together with the results of the LIVE/DEAD assay showing a homogenous distribution of viable cells across the gel volume on day 14 (Figure S2) suggest that there was no significant limitation in the nutrient supply, even to the central part of the gels.

As expected, proliferation was suppressed in all hydrogels in the CM (Figure 4B), and the number of cells slightly decreased 

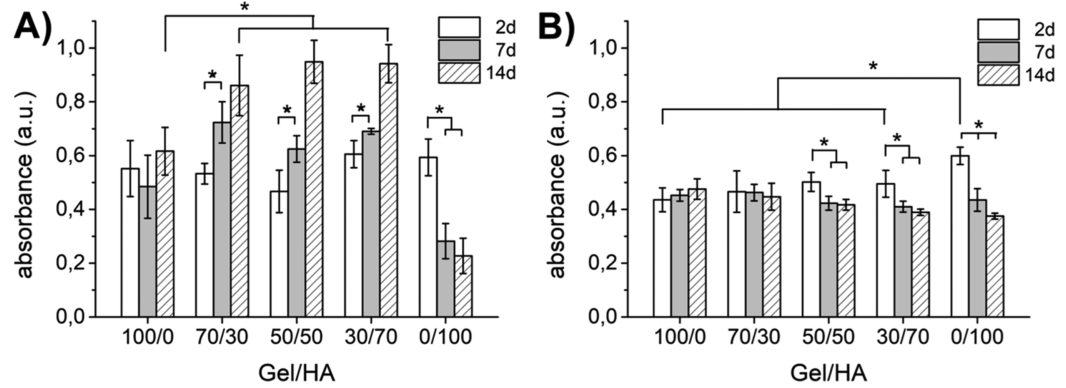

Figure 4. Cell proliferation in Gel-HA hydrogels measured by the MTS assay in the GM (A) and CM (B). The samples were compared by one-way analysis of variance (ANOVA), and statistical differences at $p<0.05$ are denoted by ${ }^{*}$, both in each Gel-HA proportion at the different time points and among the different Gel-HA compositions at the same time point.

with the culture time for those hydrogels rich in HA (50/50, $30 / 70$, and $0 / 100)$.

2.4. hMSCs Differentiation. hMSCs were encapsulated in the hydrogels to determine whether spontaneous differentiation toward specific lineages (myogenic, adipogenic, osteogenic, and chondrogenic) can occur in the absence of a differentiation medium.

Immunofluorescence images after 14 days of culture for the adipogenic marker lipoproteinlipase (LPL), osteogenic marker RUNX2, and myogenic marker MF20 in Figure 5 suggest that no differentiation toward these cell lineages could be seen in the GM in any of the Gel-HA hydrogels.

Aggrecan was studied as a well-accepted marker for chondrogenic differentiation. Aggrecan fluorescence staining was performed for all Gel-HA compositions both in the GM (Figure 6) and CM (Figure S4.1) after 14 days of culture. MSCs cultured in Gel showed a negligible expression of aggrecan, whereas the presence of HA stimulates the expression of this marker for all compositions. ${ }^{35}$

As the hydrogel mixtures and HA seemed to promote chondrogenic phenotype, other chondrogenic markers were analyzed to further study these hydrogels: SOX-9 and alcian blue staining and gene expression for collagen type II in the $\mathrm{GM}$ and $\mathrm{CM}$.

Figure 6 shows SOX-9 for cells cultured in all Gel-HA compositions in the GM (those in the CM are represented in Figure S4.2), confirming the positive results of aggrecan staining.

Alcian blue histologies are depicted in Figure 7, where glycosaminoglycans (GAGs) are stained in blue, cells are stained in red, and the background is pink or becomes purple when the quantity of HA is increased (background of the different hydrogel compositions without cells can be seen in the figure as the acellular control). As expected, dark blue staining of cells in the CM shows the presence of GAGs in all hydrogels. In the Gel hydrogel, the blue color appears less dark and more spread than in the other gels, which could be caused by better cell adhesion associated with a higher Gel degradation rate that increases the cells' capacity to synthesize the ECM (including GAGs) into larger areas. For the cells cultured in the GM, images obtained for the Gel hydrogel did not contain any blue, indicating that no GAGs were synthesized under this condition. In the case of Gel-HA and pure HA, blue areas were observed around the cells (white arrows in Figure 7), confirming the presence of GAGs in these gels.

Expression of collagen type II encoded by COL2A1 gene determined by quantitative polymerase chain reaction (qPCR) is shown in Figure 8A. The results are represented as a fold

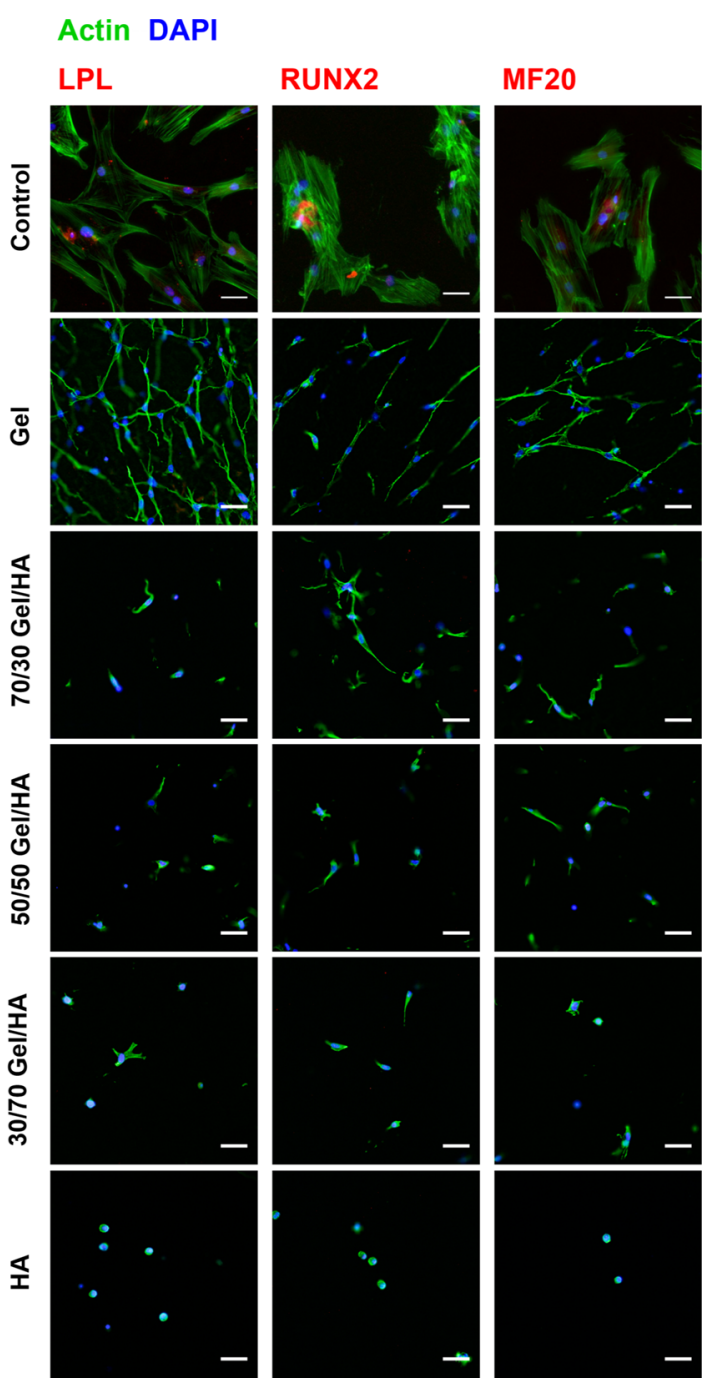

Figure 5. Immunofluorescence images for LPL, RUNX2, and MF20 of hMSCs cultured in Gel-HA hydrogels and in the GM for 14 days. Nuclei are stained with 4',6-diamidino-2-phenylindole (DAPI), cytoskeleton is stained in green, and the different antibodies are stained in red. The scale bar is $50 \mu \mathrm{m}$.

change in the expression relative to pure Gel gels in the GM, to which a value of 1 was assigned in the graph. In the GM, the cells within those hydrogels rich in HA, 30/70, and pure HA express a significantly higher amount of collagen type II than Gel in the GM. The same occurs in the CM, where the 


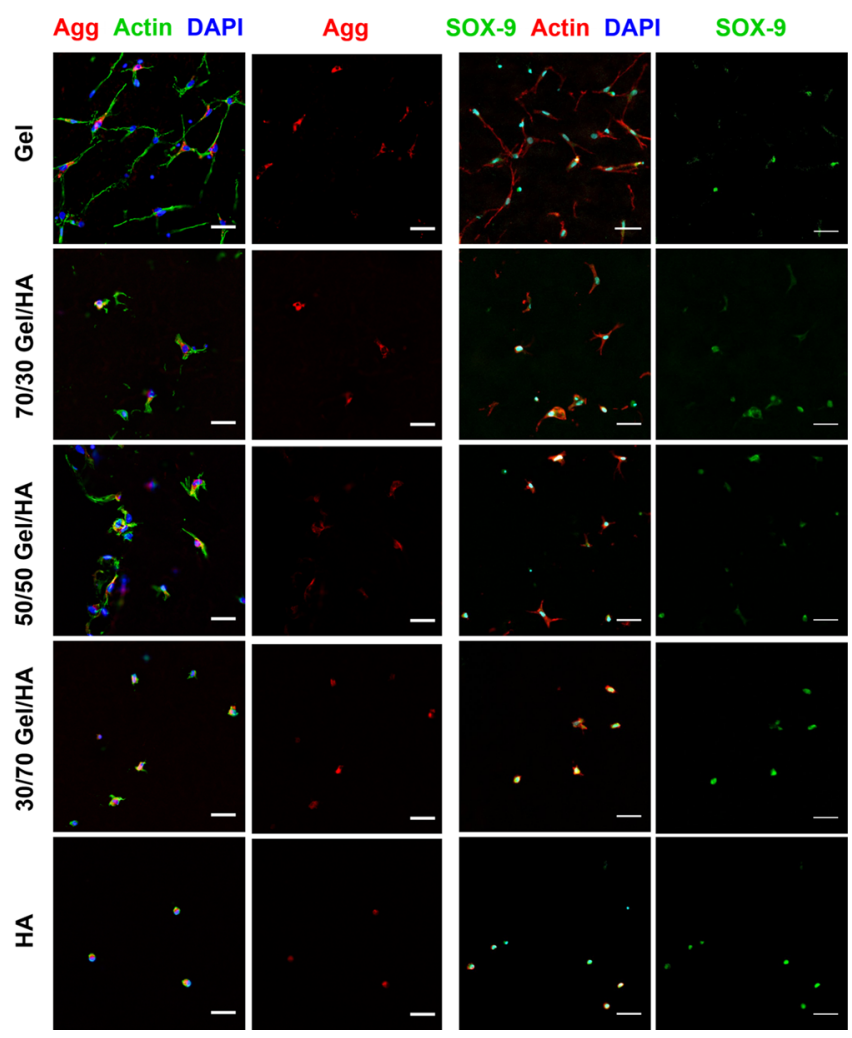

Figure 6. Immunofluorescence images for aggrecan (Agg) and SOX-9 of hMSCs cultured in Gel-HA hydrogels and in the GM for 14 days. Nuclei are stained with DAPI in blue, cytoskeleton is stained in green for Agg pictures and in red for SOX-9 pictures, aggrecan is stained in red, and SOX-9 is stained in green. The scale bar is $50 \mu \mathrm{m}$.

expression is significantly higher compared to the GM for almost all samples, except for the $70 / 30$ sample.

To quantify the levels of other chondrogenic markers already observed, immunofluorescence images as well as histological stainings were further processed to calculate the percentage of cells expressing aggrecan, SOX-9, and GAGs; the resulting graphs are shown in Figure 8.

Regarding the percentage of aggrecan expression (Figure $8 \mathrm{~B})$, again the presence of higher amounts of $\mathrm{HA}$ in the hydrogel composition increases the percentage of cells expressing aggrecan. In both CM and GM cultures, statistically significant differences were obtained between all groups, except between the 50/50 and 30/70 Gel-HA samples. The highest percentage of differentiation was obtained for pure HA hydrogel $(0 / 100)$ cultured in the GM, with $79 \%$. This value was higher than that obtained for the $0 / 100$ sample cultured in the CM (24\%), although the number of cells in this hydrogel is quite low compared with all other hydrogel compositions. GelHA 50/50 and 30/70 hybrids present similar percentages of aggrecan differentiation with $56 \%$ for the CM culture and $31 \%$ for the GM culture.

Aggrecan immunofluorescence images were also used to calculate the number of cells per area (Figure S5A), which gives us an idea of cell distribution within the different hydrogels. The number of cells per area was seen to increase with the quantity of Gel in the hydrogel, although there is a marked drop in the number of cells when HA is present in the hydrogel composition. Comparing 100/0 and 70/30 hydrogels, there is a decrease of $66 \%$ for cells in the GM and $76 \%$ for cells in the CM. No significant statistical differences were obtained

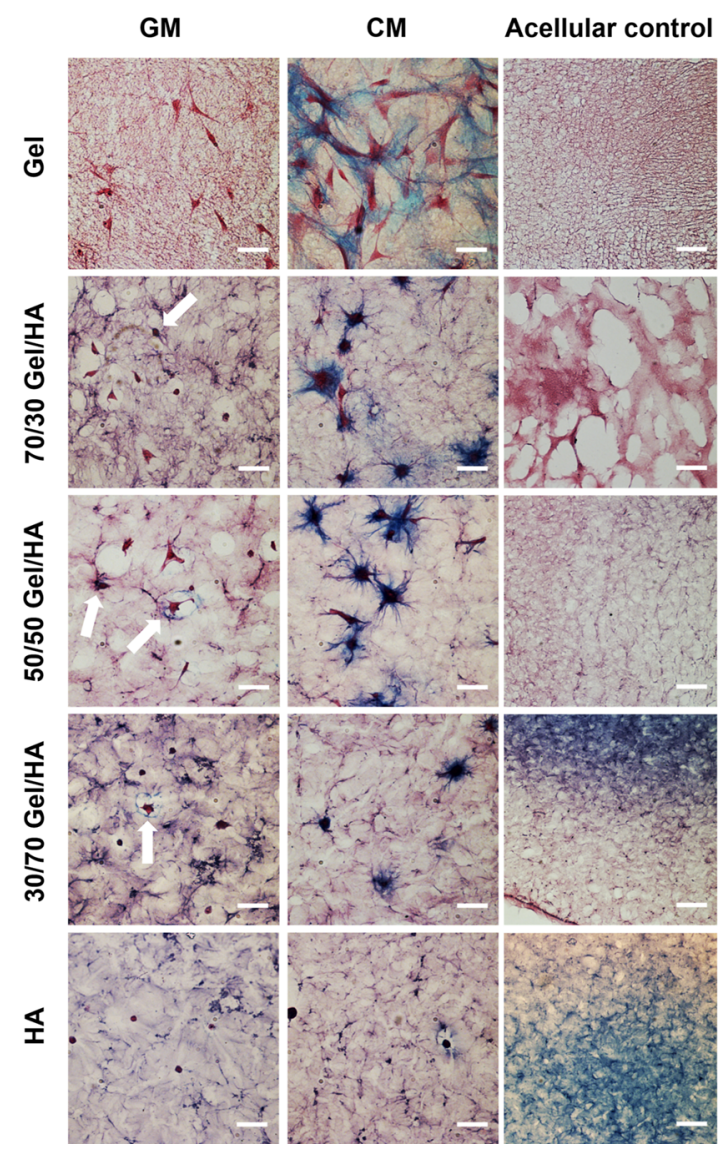

Figure 7. Alcian blue histologies with nuclear fast red staining of hMSCs cultured in Gel-HA hydrogels and in the GM and CM for 14 days with scale bar $50 \mu \mathrm{m}$. White arrows indicate cells producing GAGs for the samples cultured in the GM. Acellular controls show the alcian blue histologies for the different hydrogels without cells; here the scale bar corresponds to $100 \mu \mathrm{m}$.

between the $70 / 30$ and 50/50 hydrogels in either CM or GM or between the 50/50 and 30/70 hydrogels cultured in the GM.

As regards the percentage of SOX-9 (Figure 8C) in the CM, more than $75 \%$ of the cells are positive in all Gel-HA mixtures and HA (with no significant differences among the different hydrogels), whereas only $25 \%$ of the cells are positive in pure Gel. The percentage of positive SOX-9 cells in the GM is always higher than $45 \%$ and increases with the amount of HA in the mixtures, showing statistically significant higher values for those hydrogels rich in HA, 30/70, and HA samples.

The quantitative analysis of the alcian blue staining allowed us to calculate the percentage of cells expressing GAGs (Figure $8 \mathrm{D})$, which was complemented with the qualitative analysis determining the dark blue tone or level and the blue area around the cell (Figure S5B). In the cell-hydrogel system cultured in the GM, the percentage of cells synthesizing GAGs increased with the percentage of $\mathrm{HA}$ in the hydrogel up to values of about $80 \%$ in the $30 / 70$ mixture and HA gel. No GAG expression was detected in the case of Gel in the GM. A high percentage of cells (around 90\%) cultured in the five types of Gel-HA in the CM synthesized GAGs. In the qualitative analysis, a smaller and darker area around the cell was obtained with increased HA content in the GM, indicating a higher concentration of GAGs around the cell (Figure S5B). 

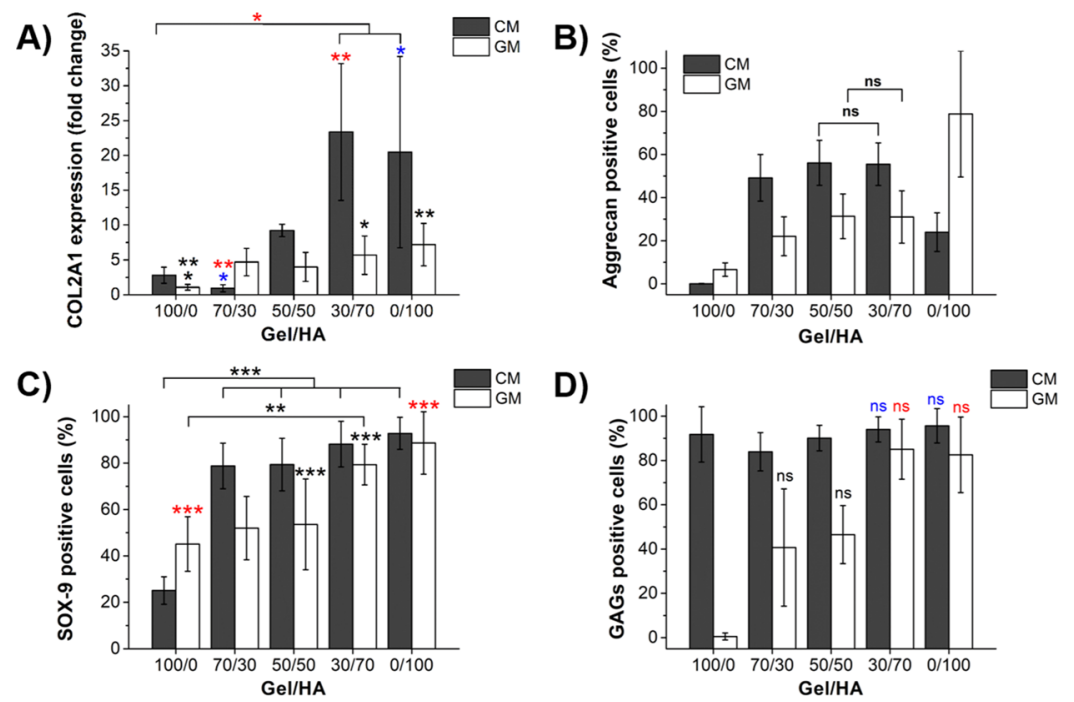

Figure 8. Quantification of the cell cultures of hMSCs encapsulated in the Gel-HA hydrogels and cultured in the GM and CM for 14 days. (A) Relative collagen type II gene expression from qPCR, represented as a change in the expression relative to Gel in the GM, to which a value of 1 was assigned in the graph. One-way ANOVA with Tukey post-test was performed to find statistical differences; $*$ for $p<0.05$, $* *$ for $p<0.01$, and $* * *$ for $p<0.005$. (B) Percentage of positive cells for aggrecan. Mann-Whitney-Wilconson test demonstrated that groups within a type of culture medium show statistically significant differences between each other, except those marked with "ns" (not significant). (C) Percentage of positive cells for SOX-9 obtained from the immunofluorescence images. One-way ANOVA with Tukey post-test was applied for samples cultured in the CM, and Kruskal-Wallis nonparametric test was applied for samples cultured in the CM. * for $p<0.05$, ** for $p<0.01$, and $* * *$ for $p<0.005$. (D) Percentage of cells expressing GAGs calculated from alcian blue images. Mann-Whitney-Wilconson test demonstrated that groups within a type of culture medium show statistically significant differences between each other, except those marked with "ns" (not significant).

\section{DISCUSSION}

Our study demonstrates that a combination of tyramine conjugates of Gel with tyramine conjugates of HA produces injectable hydrogels with an enhanced shear modulus and hydration and where chondrogenesis of MSCs without the need for growth factors is stimulated. This is demonstrated by an increased expression of aggrecan, SOX-9, collagen type II, and GAGs. The importance of having hydrogels that do not need the supplement of growth factors in tissue-engineering applications is multiple. As described in refs 23 and 36, chondrogenic growth factors can also induce osteogenesis; therapies based on them are cost inefficient and not clinically attractive; ${ }^{37}$ and the release of growth factors from hydrogels is usually not sustained unless they are chemically modified. ${ }^{20}$

Similar analogs of the ECM based on collagen and GAG have demonstrated that the range of stiffness similar to our matrices, $0.5 \mathrm{kPa}$, was optimal to direct MSCs toward chondrogenic lineage. ${ }^{22}$ However, the same compositions with higher stiffness, $1.5 \mathrm{kPa}$, upregulated the osteogenic expression. Both cases were tested in the absence of differentiation supplements. Having our composites a lower stiffness than the one described as osteogenic could be the cause of the negative RUNX2 expression in our hydrogels. MSCs myogenic induction also needs a certain mechanical stiffness (10 kPa in ref 38$)$ and usually requires the addition of several specific growth factors when MSCs are encapsulated in hydrogels. ${ }^{39}$ Although adipogenesis is promoted in softer hydrogels, MSCs in them usually need to be in very high densities, which could be the reason our hydrogels did not show LPL expression. ${ }^{40,41}$

Although pure Gel hydrogels have been proposed for cartilage tissue engineering, the adhesion of the encapsulated cells is very strong and tend to have a stretched morphology with secretion of markers that are not typical of articular cartilage, such as collagen type I and hypertrophy markers. ${ }^{35}$
From the mechanical point of view, Gel stiffness is too low ( $172 \mathrm{~Pa}$ of storage shear modulus, Figure $2 \mathrm{~A}$ ) and is not able to counterbalance the traction forces exerted by cells, resulting in a dramatic shrinkage during the in vitro culture, as previously shown in ref 25. These shortcomings and the fast degradation rate of Gel make the mixtures more attractive materials.

The chondrogenic potential of HA is well-known ${ }^{20}$ and has predominated in the hydrogel mixtures that also promoted chondrogenesis. During cell mitosis and migration, a thin pericellular layer rich in HA is secreted by cells, which mediates their detachment from the ECM and promotes cell rounding. ${ }^{42}$ In cartilage, the chondrocyte CD44 receptor interacts with secreted HA chains, keeping the cells surrounded by a gel-like environment that is crucial for maintaining the differentiated phenotype. ${ }^{43}$ However, pure HA hydrogel has some limitations because of the low or even null cell proliferation and the fact that secreted ECM tends to remain in the pericellular space and not distributed within the hydrogel. ${ }^{35}$ Our results show that although in HA, a rounded cell morphology with the expression of chondrogenic markers is obtained in the GM (Figure 6) and CM (Figures S4.1 and S4.2) (probably due to the interaction of hMSCs with the HA chains by the CD44 receptor), the number of cells is very low and decreases with the time of culture (Figure 3).

The hybrid Gel-HA matrices mimic the composition of the ECM and combine the cell adhesive chains of Gel (containing RGD sequences) with HA chains that are more rigid than Gel, provide stiffness, have a lower degradation rate than Gel, and induce chondrogenesis. The in vitro cultures in the GM show that the percentage of cells synthesizing aggrecan, SOX-9, collagen type II, and GAGs increases with the percentage of HA in the hydrogel. Although pure HA seems to promote the chondrogenic phenotype, it is not the best matrix for cartilage tissue engineering as cell adhesion to this material is very poor, and it also does not promote cell proliferation. $50 / 50$ or $30 / 70$ 
Gel-HA mixtures seem to be better candidates for the encapsulation of hMSCs as they allow both cell adhesion and proliferation and still benefit from the presence of HA, enhancing cell differentiation into the chondrogenic phenotype. Our results are consistent with others reporting that the incorporation of $\mathrm{HA}$ in hydrogels promotes chondrogenic differentiation. ${ }^{19,28}$ In particular, Levett et al. ${ }^{19}$ demonstrated that dedifferentiated chondrocytes encapsulated in threedimensional Gel-HA hydrogels containing small amounts of HA were able to redifferentiate to chondrocytes.

As Gel resulted in a higher substitution degree of tyramine than $\mathrm{HA},{ }^{25} \mathrm{Gel}$ network is more cross-linked than HA, which is consistent with its lower swelling capacity in comparison to HA (Figure 2D and Table 2), being the mixtures between the values of pure networks. The apparent cross-linking density was calculated elsewhere ${ }^{25}$ and was higher for Gel $\left(1.95 \mathrm{~mol} / \mathrm{m}^{3}\right)$ than for HA $\left(0.69 \mathrm{~mol} / \mathrm{m}^{3}\right)$, having the mixtures cross-linking densities within these values (Table 2). The apparent crosslinking density of the degraded hydrogels (20-30\% mass loss) decreased for Gel and Gel-HA mixtures, obtaining a decrease from 1.95 to $0.68 \mathrm{~mol} / \mathrm{m}^{3}$ for pure Gel hydrogel (Table 2), as usually occurs in bulk homogeneous degradation of networks. ${ }^{27}$ Internal hydrogel degradation creates a chain cleavage in the peptide bond (for Gel) ${ }^{6}$ and in the $\beta-1-4$ glycosidic linkages (for HA), ${ }^{27}$ causing reduced hydrogel cross-linking that creates loosened networks of higher mesh size with more hydroxyl groups or bigger pores capable of absorbing more water. Also, because Gel degrades first (Figure 2C), it will leave gaps or small pores that will increase enzyme diffusion and hydrogel degradation. On the other hand, no change in EWC was obtained after 30\% degradation in the HA hydrogel (Figure 2D). As previously reported, ${ }^{44}$ the difficulty of hyaluronidase diffusion inside the pure HA hydrogel provokes surface degradation causing hydrogel mass loss without changing the apparent cross-linking density, which seems to increase from 0.69 to $1.02 \mathrm{~mol} / \mathrm{m}^{3}$ after $20-30 \%$ degradation (see Table 2) but with no significant difference from the nondegraded sample (Figure 2C).

The mechanical stiffness of hydrogels depends on the crosslinking degree, the water content, the chemical composition, the rigidity of the chains, and the water permeability coefficient. Because HA has a lower cross-linking degree, it would be expected that hydrogels with a higher HA content would have a lower shear modulus. However, this is not the case because hydrogels with a higher HA content have a higher mechanical modulus and a higher swelling degree (Figure 2A,D). The rigidity of $\mathrm{HA}$ chains in water and the lower hydraulic permeability of water from HA would explain an increase in the storage modulus of the hydrogels from $172 \mathrm{~Pa}$ for pure Gel to $789 \mathrm{~Pa}$ for pure HA (Figure $2 \mathrm{~A})^{21,25}$ as the ratio of HA in the mixtures increases.

Overall, the 30/70 Gel-HA mixture shows characteristics that are most suitable for regenerative therapies in cartilage damage. It is the composite that had the lowest degradation rate but still presented bulk degradation demonstrated by the lower cross-linking density after $20-30 \%$ degradation. This means that those spaces left in the hydrogel mesh after degradation would allow spreading of secreted ECM, which would not occur in pure HA where surface degradation was inferred from the swelling results after degradation. This mixture still benefits from integrin adhesion cues provided by Gel that promotes cytoskeleton development and cell proliferation. In addition, the high HA content allowed to increase the mechanical stiffness up to $690 \mathrm{~Pa}$, very close to that of pure $\mathrm{HA}$, confirming the stability of the network provided by $\mathrm{HA}$ in the mixtures, and most importantly, it has been the composite that shows the greatest benefit from the chondrogenic HA biochemical cues in the absence of differentiation supplements. 30/70 Gel-HA hydrogel is the only mixture that showed a significant difference of collagen type II expression and SOX-9 positive cell percentage compared to Gel in the GM. Furthermore, aggrecan and GAGs positive cell percentage of the $30 / 70$ composite is the highest among the mixtures and equal to the percentages found in pure HA. The null proliferative potential, poor cell cytoskeleton development, and surface degradation profile could be sufficient arguments to defend that the HA hydrogel needs the combination with small amounts of cell adhesive protein to optimize its chondrogenic potential, whereas pure Gel results in insufficient mechanical stiffness and enhanced cell adhesion inhibiting MSC chondrogenesis in three-dimension in the absence of specific growth factors.

\section{CONCLUSIONS}

Enzymatically cross-linked injectable Gel and HA hydrogel hybrids show a high potential as systems for the regeneration of articular cartilage as chondrogenic differentiation is promoted even in the GM. Including HA in the mixtures provides better mechanical properties than pure Gel and adds stability in terms of degradability. Moreover, the presence of HA stimulates aggrecan, SOX-9, collagen type II, and GAG synthesis. Gel is needed in the hybrids to improve cell adhesion and for their retention/proliferation over an extended period as very few cells are found in the pure HA hydrogels.

\section{EXPERIMENTAL SECTION}

5.1. Materials. HA sodium salt from Streptococcus equi and Gel from porcine skin (gel strength 300, type A) were purchased from Sigma-Aldrich (USA). Sodium chloride (synthesis grade) and potassium dihydrogen phosphate (extra pure) were purchased from Scharlab (Spain). N-(3-Dimethylaminopropyl)- $N^{\prime}$-ethylcarbodiimide hydrochloride (EDC) was supplied by Iris Biotech $\mathrm{GmbH}$ (Germany). All other reagents used in the Gel-HA synthesis and characterization were purchased from Sigma-Aldrich.

Calcium-free Krebs Ringer buffer (CF-KRB) solution was prepared with $115 \mathrm{mM}$ sodium chloride, $5 \mathrm{mM}$ potassium chloride, $1 \mathrm{mM}$ potassium dihydrogen phosphate, and $25 \mathrm{mM}$ 4-(2-hydroxyethyl)piperazine-1-ethanesulphonic acid.

For cell culture experiments, the human bone marrow mesenchymal stem cells (BM-hMSCs) were purchased from a commercial lineage (PromoCell, Germany). Primary antibodies against aggrecan (mouse), SOX-9 (mouse), RUNX2 (rabbit), and LPL (rabbit) were purchased from Santa Cruz Biotechnologies (USA). Mouse primary antibody for myosin (MF-20b, $800 \mu \mathrm{g} / \mathrm{mL}$ ) was purchased from Developmental Studies Hybridoma Bank (DSHB, USA). Secondary antibodies rabbit anti-mouse IgG $\mathrm{Cy} 3$ and goat anti-rabbit IgG Cy3 were purchased from Jackson Immunoresearch (USA), and donkey anti-mouse AF 488 antibody was purchased from Life Technologies (UK). Fungizone, insulin-transferrin-selenium-X (ITS-X), phosphate buffered saline (PBS), DPBS were purchased from Gibco, ThermoFisher Scientific (USA). BODIPY phallacidin and rhodamine phalloidin were purchased from Life Technologies (USA). TGF- $\beta 3$ was purchased from 
R\&D Systems (USA). Embedding medium for cryotomy (OCT compound) was purchased from VWR (USA). VECTASHIELD with DAPI was purchased from Vector Laboratories (USA). DPX mounting medium was purchased from Fisher Scientific (USA). Alcian blue 8GX and nuclear fast red (94\%, pure) were purchased from Acros Organics (USA). RNeasy Micro Kit, QuantiTect Reverse Transcription Kit and QuantiFast SYBR Green PCR Kit were purchased from Qiagen. SsoAdvanced PreAmp Supermix was bought from Bio-Rad, and primers for collagen type II $\mathrm{qPCR}$ were ordered from Invitrogen. All other reagents were purchased from SigmaAldrich.

5.2. Gel and HA Hydrogel Synthesis. Hydrogel mixtures with different proportions of Gel and HA were obtained by enzymatically cross-linking their tyramine conjugates in the same way as described in ref 25 .

For tyramine grafting onto Gel, 2\% (w/v) Gel in $50 \mathrm{mM} 2$ ( $N$-morpholino)ethanesulfonic acid ( $>99 \%$, MES) was dissolved at $60{ }^{\circ} \mathrm{C}$ under stirring. Then tyramine hydrochloride (98\%, Tyr) was added (2:1 Tyr/COOH molar ratio) and stirred for $20 \mathrm{~min}$ at room temperature (RT). The $\mathrm{pH}$ was adjusted to 6 and $N$-hydroxysucciniamide (NHS) (98\%) was added and stirred. EDC was then added, and the mixture was stirred for $24 \mathrm{~h}$ at $37{ }^{\circ} \mathrm{C}$. The molar ratios were 2:1 for EDC/ $\mathrm{COOH}$ and 1:10 for NHS/EDC. The solution was then dialyzed [dialysis tubing 12400 molecular weight cutoff (MWCO)] against deionized water for $48 \mathrm{~h}$. Finally, the modified Gel was lyophilized in a LyoQuest freeze dryer (Telstar Life Science Solutions, Japan).

Before tyramine bonding with $\mathrm{HA}$, the molecular weight of $\mathrm{HA}$ was reduced from $1.06 \mathrm{MDa}$ to $\sim 320000 \mathrm{Da}$ by acidic degradation. ${ }^{25}$ For the tyramine grafting, $0.5 \mathrm{w} / \mathrm{v} \%$ of the lowmolecular-weight $\mathrm{HA}$ was dissolved in $150 \mathrm{mM} \mathrm{NaCl}, 276 \mathrm{mM}$ MES, and $75 \mathrm{mM} \mathrm{NaOH}$ at $\mathrm{pH}$ 5.75. Subsequently, tyramine hydrochloride was added (2:1 Tyr/COOH molar ratio) and stirred until dissolution, and the $\mathrm{pH}$ was adjusted at 5.75 . Afterward, EDC (1:1 EDC/COOH molar ratio) and $\mathrm{NHS}$ (1:10 NHS/EDC molar ratio) were added and stirred for $24 \mathrm{~h}$ until the reaction was completed. Finally, dialysis (dialysis tubing of $3500 \mathrm{MWCO}$ ) against $150 \mathrm{mM} \mathrm{NaCl}$ was performed for $24 \mathrm{~h}$ and against deionized water another $24 \mathrm{~h}$, with three changes of dialysis solution each day. The modified HA was dried in the lyophilizer.

To prepare the hydrogels, solutions of pure tyraminemodified Gel and HA at $2 \mathrm{w} / \mathrm{v} \%$ in CF-KRB were made at 37 ${ }^{\circ} \mathrm{C}$. For proper HA dissolution, the solution was prepared 1 day early and left to dissolve at $4{ }^{\circ} \mathrm{C}$ for $24 \mathrm{~h}$. Gel solution $(2 \mathrm{w} / \mathrm{v}$ $\%)$ was fully dissolved after $30 \mathrm{~min}$ at $37{ }^{\circ} \mathrm{C}$. Different volumetric proportions of Gel-HA were obtained (100/0, 70/ $30,50 / 50,30 / 70$, and $0 / 100)$. The hydrogels were formed with $80 \mathrm{v} / \mathrm{v} \%$ of the $2 \mathrm{w} / \mathrm{v} \%$ Gel-HA mixtures, $10 \mathrm{v} / \mathrm{v} \% \mathrm{HRP}$ at $12.5 \mathrm{U} / \mathrm{mL}(1.25 \mathrm{U} / \mathrm{mL}$ in the final volume), and $10 \mathrm{v} / \mathrm{v} \%$ $\mathrm{H}_{2} \mathrm{O}_{2} 20 \mathrm{mM}$ ( $2 \mathrm{mM}$ in the final volume) after few minutes of adding the peroxide.

5.3. Mechanical Characterization of Hydrogels by Rheology. Shear deformation rheological experiments were performed on a strain-controlled AR-2000ex rheometer (TA Instruments). A solvent trap geometry of parallel plates (made of nonporous stainless steel, diameter $=20 \mathrm{~mm}$ ) was used to reduce the solvent loss during the experiment. The gap between the plates was around $1200 \mu \mathrm{m}$. The sample temperature was controlled and maintained by a Peltier device at $37{ }^{\circ} \mathrm{C}$. The mixtures of Gel, HA, and the enzyme (HRP) were arranged on the plate at $37^{\circ} \mathrm{C}$ and cross-linked by adding the correct amount of $\mathrm{H}_{2} \mathrm{O}_{2}$. After $20 \mathrm{~min}$, the samples had been cross-linked, and two different measurements were performed. First, the range of strain amplitudes at which the gels exhibit a linear region of viscoelasticity was determined. A dynamic strain sweep (with amplitudes ranging between $0.01 \%$ and $15 \%$ ) was carried out at a frequency of $1 \mathrm{~Hz}$ to measure the dynamic shear modulus as a function of strain. Second, to determine the dependence of the dynamic shear modulus and loss factor on the frequency, a dynamic frequency sweep test was performed between 0.1 and $10 \mathrm{~Hz}$ at $1 \%$ strain, corresponding to the hydrogel linear region.

The following data were obtained from the rheological measurements: storage modulus $\left(G^{\prime}\right)$, loss modulus $\left(G^{\prime \prime}\right)$, complex modulus magnitude $\left[\left|G^{*}\right|\right.$, a measure of the hydrogel stiffness: $\left.\left|G^{*}\right|^{2}=\left(G^{\prime}\right)^{2}+\left(G^{\prime \prime}\right)^{2}\right]$, and loss factor $\left[\tan \delta \equiv\left(G^{\prime \prime}\right) /\right.$ $\left(G^{\prime}\right)$, a measure of the internal energy dissipation, where $\delta$ is the phase angle between the applied stimulus and the corresponding response] as a function of the strain amplitude or frequency.

5.4. Enzymatic Degradation Study. After the hydrogel synthesis, the hydrogels were left overnight in DPBS with 0.02 $\mathrm{w} / \mathrm{v} \%$ sodium azide to remove unreacted substances and reach equilibrium swelling. The in vitro degradation of Gel-HA hydrogels was subsequently performed by incubating the hydrogels with hyaluronidase and collagenase at $37{ }^{\circ} \mathrm{C}$. Cylindrical samples $(7 \mathrm{~mm}$ diameter and $280 \mu \mathrm{L}$ volume) were incubated in $10 \mathrm{U} / \mathrm{mL}$ of hyaluronidase (type IV-S from bovine testes, Sigma-Aldrich) and $3 \mathrm{U} / \mathrm{mL}$ of collagenase (type IA from Clostridium histolyticum, Sigma-Aldrich) solutions in DPBS with $0.5 \mathrm{w} / \mathrm{v} \%$ sodium azide at $37{ }^{\circ} \mathrm{C}$. Five replicates were conducted for each composition and time point.

Degradation was followed by mass loss of the hydrogel as a function of time. The initial swollen mass $\left(m_{\mathrm{s}, t_{0}}\right)$ was noted and measured at different time points $\left(m_{\mathrm{s}, t}\right)$, which provided mass loss by eq 1

$$
\% \text { mass loss }=\frac{m_{\mathrm{s}, t_{0}}-m_{\mathrm{s}, t}}{m_{\mathrm{s}, t_{0}}} \times 100
$$

The EWC of the hydrogel swollen in DPBS with $0.02 \mathrm{w} / \mathrm{v} \%$ sodium azide overnight, which was used for comparison after formation after reaching 20-30\% degradation, was obtained by eq 2

$$
\% \mathrm{EWC}=\frac{m_{\mathrm{s}, \mathrm{d}}-m_{\mathrm{d}, \mathrm{d}}}{m_{\mathrm{d}, \mathrm{d}}} \times 100
$$

where $m_{\mathrm{s}, \mathrm{d}}$ is the swollen mass at $20-30 \%$ degradation and $m_{\mathrm{d}, \mathrm{d}}$ is the dried mass at $20-30 \%$ degradation.

5.5. Cell Culture in Gel-HA Hydrogels. BM-hMSCs were expanded in the presence of a GM consisting of Dulbecco's modified Eagle's medium (DMEM), a high glucose-based medium with $0.4 \%$ penicillin/streptomycin solution (stock solution, $10000 \mathrm{U} / \mathrm{mL}$ penicillin and $10 \mathrm{mg} /$ $\mathrm{mL}$ streptomycin), $1 \mathrm{mM}$ L-glutamine, $0.05 \%$ FUNGIZONE (stock solution at $250 \mu \mathrm{g} / \mathrm{mL}$ ), $100 \mu \mathrm{M}$ sodium pyruvate, and $10 \%$ fetal bovine serum (FBS) at $37{ }^{\circ} \mathrm{C}$ and $5 \% \mathrm{CO}_{2}$ in an incubator.

Gel (2 w/v \%) and HA ( $2 \mathrm{w} / \mathrm{v} \%)$ solutions were prepared by dissolving the lyophilized powder in DMEM with $1 \% \mathrm{P} / \mathrm{S}$, $24 \mathrm{~h}$ at $4{ }^{\circ} \mathrm{C}$ for HA and $30 \mathrm{~min}$ at $37^{\circ} \mathrm{C}$ for Gel. HRP solution $(12.5 \mathrm{U} / \mathrm{mL})$ was then added to the prepared solutions at a volume ratio of $10 / 80(\mathrm{~mL}$ of $\mathrm{HRP} / \mathrm{mL}$ Gel or HA solution), 
and the obtained mixture was filtered through a $0.22 \mu \mathrm{m}$ syringe filter for sterilization. Then, solutions in different proportions $(100 / 0,70 / 30,50 / 50,30 / 70$, and $0 / 100 \mathrm{v} / \mathrm{v})$ of Gel + HRP and HA + HRP were prepared.

BM-hMSCs were detached from the flask using trypsin ethylenediaminetetraacetic acid, neutralized with the GM, centrifuged at $1400 \mathrm{rpm}$ for $5 \mathrm{~min}$, resuspended in the GM, and counted with a hemocytometer. The required amount of BM-hMSCs (passage 6-7) cells $\left(1 \times 10^{6}\right.$ cells $\left./ \mathrm{mL}\right)$ was added to each Gel-HA mixture. Finally, $45 \mu \mathrm{L}$ of the Gel-HA cell suspension was cross-linked with $5 \mu \mathrm{L}$ of $20 \mathrm{mM} \mathrm{H}_{2} \mathrm{O}_{2}$ on each well of the cell culture plate and left in an incubator at $37{ }^{\circ} \mathrm{C}$ and $5 \% \mathrm{CO}_{2}$ for $30 \mathrm{~min}$ to ensure hydrogel cross-linking. Finally, triplicates of each composition were cultured in the GM and CM, the latter composed of GM without FBS and with 100 $\mathrm{nM}$ dexamethasone, $1 \%$ ITS-X, $50 \mu \mathrm{g} / \mathrm{mL}$ ascorbic 2phosphate, $40 \mu \mathrm{g} / \mathrm{mL}$ L-proline, and $10 \mathrm{ng} / \mathrm{mL}$ TGF- $\beta 3 .^{26}$ The hydrogels formed a drop of about $7 \mathrm{~mm}$ diameter at the bottom of the cell culture wells (nonadhesive wells were used to prevent cell interaction with them), which means that the maximum thickness of the hydrogels was $3.5 \mathrm{~mm}$. Cell culture was followed for 14 days, and the cell medium was changed every 2 days.

5.6. Cell Proliferation Assay (MTS). Cell proliferation was studied by analyzing the cell viability on days 2, 7, and 14 of culture using the MTS assay, following manufacturer instructions. Briefly, the cell-cultured samples (four replicates) were moved to a new cell culture plate and incubated with a fresh culture medium without phenol red or FBS but containing the MTS reagent (ratio 5:1) at $37{ }^{\circ} \mathrm{C}$ for $2 \mathrm{~h}$ in the dark. Thereafter, the absorbance of $100 \mu \mathrm{L}$ of supernatant transferred to a new cell culture plate was measured at 490 and $690 \mathrm{~nm}$ with an Infinite 200 PRO plate reader (Tecan, Switzerland). Absorbance at $490 \mathrm{~nm}$ is proportional to the number of viable cells in each sample, whereas the absorbance at $690 \mathrm{~nm}$ is used to subtract the potential background signal of small pieces of hydrogels inside each well.

5.7. Immunofluorescence Study. After 14 days of culture, the samples were washed with PBS, fixed with $4 \%$ formaldehyde for $15 \mathrm{~min}$, and washed again with PBS to remove the formaldehyde solution. After the fixing step, the samples were soaked overnight in $30 \mathrm{w} / \mathrm{v} \%$ sucrose in DPBS, embedded in OCT, and frozen with liquid nitrogen. Finally, $40 \mu \mathrm{m}$ sections were cut out with a Leica CM 1860 UV cryostat.

Gel-HA gel sections cultured in the GM and CM were immunostained for aggrecan and SOX-9, two characteristic components of articular cartilage. First, the sections on the slides were washed and rehydrated with PBS, permeabilized with $0.1 \%$ Triton X-100 in PBS for 20 min at RT and given two 5 min washes with PBS. The blocking buffer, formed by $1 \%$ bovine serum albumin (BSA) solution in PBS, was then added for $1 \mathrm{~h}$ at RT, and two washes with PBS were performed. Primary antibodies were diluted 1:100 in the blocking buffer, and two sets of samples were separately incubated with aggrecan and SOX-9 antibody solutions for $1 \mathrm{~h}$ at RT. Then, the samples were washed and incubated with donkey anti-rabbit rhodamine secondary antibody solution for aggrecan staining and with the donkey antimouse AF 488 secondary antibody for SOX-9 for $1 \mathrm{~h}$ at RT, both secondaries diluted 1:200 in the blocking buffer. Finally, two washes with PBS for 10 min were carried out; actin was stained with BODIPY FL phallacidin (aggrecan) or with rhodamine phalloidin (SOX-9) for $30 \mathrm{~min}$ at RT (both diluted 1:100 in PBS). The samples were washed, and the slides were mounted with VECTASHIELD with DAPI.

For Gel-HA samples cultured in the GM, other antibodies, characteristic of other cell lineages, were tested to determine whether the BM-hMSCs in these types of gels tend to differentiate into one or the other cell lineage. For this, the hydrogel sections were washed and rehydrated with PBS, permeabilized with $0.5 \%$ Triton X-100 in PBS at RT, rinsed with PBS twice for 5 minutes, blocked in 1\% BSA/0.1\% Triton $\mathrm{X}-100$ in PBS for $1 \mathrm{~h}$ at RT, and rinsed with PBS. The following primary antibodies were then incubated in the blocking buffer for $1 \mathrm{~h}$ at RT: rabbit polyclonal RUNX2, rabbit polyclonal LPL, and mouse monoclonal MF-20. Two $5 \mathrm{~min}$ washes were performed, and the secondary antibody $\mathrm{Cy} 3$ antimouse or antirabbit was incubated, according to the primary antibody used, at 1:200 in the blocking buffer for $1 \mathrm{~h}$ at RT. After two washes with PBS, actin was stained with BODIPY phallacidin 1:100 in PBS and washed twice for $10 \mathrm{~min}$, and the stained sections were mounted in VECTASHIELD with DAPI.

5.8. Alcian Blue Histochemistry. Gel-HA hydrogels without cells, as controls, and samples cultured for 14 days in the GM or CM were stained with alcian blue to localize GAGs within the hydrogels. The hydrogel section slides were rehydrated by washing with $\mathrm{PBS}$ twice for $5 \mathrm{~min}$ and then incubated in $1 \%$ alcian blue in $0.1 \mathrm{~N} \mathrm{HCl}$ at $\mathrm{pH} 1$ for $30 \mathrm{~min}$ to stain sulfated GAGs, rinsed with tap water and distilled water, and counterstained with $0.1 \%$ nuclear fast red for $5 \mathrm{~min}$. The slides with the sections were then rinsed in tap water, rinsed with distilled water, and dehydrated with increasing ethanol solutions (70 and 90\%) and xylene for 1 min each wash. Finally, the sections were mounted with the DPX mounting medium. GAGs positive staining was documented by optical microscopy using bright-field illumination.

5.9. Gene Expression for Collagen Type II. RNA was extracted from the gels after 14 days of incubation using an RNeasy Micro kit; briefly, samples were washed with PBS, then lysed/homogenized, mixed with $70 \%$ ethanol, and loaded onto a microcolumn and spun; then the columns were washed, DNase treated, washed again, and finally eluted in sterile water. After RNA concentration measurement and RNA quality check on NanoDrop, cDNA was synthetized; preamplification step was performed using a ProFlex thermocycler (Applied Biosciences), and finally, qPCR was run on a CFX-96 thermocycler (Bio-Rad) using GAPDH as the housekeeping gene. The primer sequences were as follows: COL2A1-forward 5'-GGC AAT AGC AGG TTC ACG TAC A-3'; COL2A1revers 5'-CGA TAA CAG TCT TGC CCC ACT T-3'; GAPDH-forward 5'-AGG TCG GTG TGA ACG GAT TTG3'; GAPDH-revers 5'-TGT AGA CCA TGT AGT TGA GGT CA-3'. Results were analyzed with CFX Manager software.

5.10. Statistical Analysis. For the statistical studies, either Statgraphics or GraphPad Prism5 software was used. MannWhitney-Wilcoxon test, one-way ANOVA with Tukey posttest, Kruskal-Wallis nonparametric test with Dunn's post-test, or unpaired two-tailed $t$-test was performed where applicable.

\section{ASSOCIATED CONTENT}

\section{Supporting Information}

The Supporting Information is available free of charge on the ACS Publications website at DOI: 10.1021/acsomega.7b01303.

Graph for the complex shear modulus of the samples as a function of strain, images for the LIVE/DEAD assay, 
optical microscope pictures of cells cultured in the hydrogels for 2, 7, and 14 days, and immunofluorescence images for aggrecan and SOX-9 after 14 days of culture in the $\mathrm{CM}(\mathrm{PDF})$

\section{AUTHOR INFORMATION}

\section{Corresponding Authors}

*E-mail: Manuel.Salmeron-Sanchez@glasgow.ac.uk (M.S.-S.).

*E-mail: ggallego@ter.upv.es (G.G.F.).

\section{ORCID}

Sara Poveda-Reyes: 0000-0002-4869-5134

Manuel Salmerón-Sánchez: 0000-0002-8112-2100

Gloria Gallego Ferrer: 0000-0002-2428-0903

\section{Author Contributions}

The manuscript was written through contributions of all authors. All authors have given approval to the final version of the manuscript. V.M. and S.P.-R. contributed equally.

\section{Notes}

The authors declare no competing financial interest.

Data availability: All the original data related to this article are within the depository of the University of Glasgow with DOI: $10.5525 /$ gla.researchdata.495.

\section{ACKNOWLEDGMENTS}

The authors are grateful for the financial support received from the Spanish Ministry through the MAT2016-76039-C4-1-R project (including the FEDER financial support), the BES2011-046144, and the EEBB-I-14-08725 grants. The CIBERBBN initiative is funded by the VI National R\&D\&I Plan 2008-2011, Iniciativa Ingenio 2010, Consolider Program. CIBER actions are financed by the Instituto de Salud Carlos III with assistance from the European Regional Development Fund. M.S.-S. acknowledges the European Research Council (ERC-HealInSynergy 306990) and the UK Engineering and Physical Sciences Research Council (EPSRC-EP/P001114/ 1).

\section{ABBREVIATIONS}

$\mathrm{BA}$, blue area; BL, blue level; BM-hMSCs, bone marrow human mesenchymal stem cells; CF-KRB, calcium free Krebs Ringer buffer; CM, chondrogenic medium; DPBS, Dulbecco's phosphate buffered saline; ECM, extracellular matrix; EDC, $N$-(3-dimethylaminopropyl)- $N^{\prime}$-ethylcarbodiimide hydrochloride; EWC, equilibrium water content; GAGs, glycosaminoglycans; Gel, gelatin; Gel-HA, gelatin hyaluronic acid mixtures; GM, growth medium; HA, hyaluronic acid; HEPES, 4-(2hydroxyethyl)piperazine-1-ethanesulphonic acid; hMSCs, human mesenchymal stem cells; HRP, horseradish peroxidase; ITS-X, insulin-transferrin-selenium-X; LPL, lipoproteinlipase; MES, 2-( $N$-morpholino)ethanesulfonic acid; MF-20, sarcomeric myosin; MTS, (3-(4,5-dimethylthiazol-2-yl)-5-(3-carboxymethoxyphenyl)-2-(4-sulfophenyl)-2H-tetrazolium) proliferation assay; NHS, N-hydroxysuccinimide; NP, nucleus pulposus; OCT, embedding medium for cryotomy; PBS, phosphate buffered saline; qPCR, quantitative polymerase chain reaction; RT, room temperature; SOX-9, Sry-type high mobility group box transcription factor 9; Tyr, tyramine hydrochloride

\section{REFERENCES}

(1) Wang, T.-W.; et al. The effect of gelatin-chondroitin sulfatehyaluronic acid skin substitute on wound healing in SCID mice. Biomaterials 2006, 27, 5689-5697.

(2) Bhat, S.; Kumar, A. Cell proliferation on three-dimensional chitosan-agarose-gelatin cryogel scaffolds for tissue engineering applications. J. Biosci. Bioeng. 2012, 114, 663-670.

(3) Cohen, N. P.; Foster, R. J.; Mow, V. C. Composition and Dynamics of Articular Cartilage: Structure, Function, and Maintaining Healthy State. J. Orthop. Sports Phys. Ther. 1998, 28, 203-215.

(4) Spiller, K. L.; Maher, S. A.; Lowman, A. M. Hydrogels for the Repair of Articular Cartilage Defects. Tissue Eng., Part B 2011, 17, 281-299.

(5) Frantz, C.; Stewart, K. M.; Weaver, V. M. The extracellular matrix at a glance. J. Cell Sci. 2010, 123, 4195-4200.

(6) Choi, Y. S.; et al. Studies on gelatin-containing artificial skin: II. Preparation and characterization of cross-linked gelatin-hyaluronate sponge. J. Biomed. Mater. Res. 1999, 48, 631-639.

(7) Shu, X. Z.; Liu, Y.; Palumbo, F.; Prestwich, G. D. Disulfidecrosslinked hyaluronan-gelatin hydrogel films: a covalent mimic of the extracellular matrix for in vitro cell growth. Biomaterials 2003, 24, 3825-3834.

(8) Slaughter, B. B.; et al. Hydrogels in Regenerative Medicine. Adv. Mater. 2009, 21, 3307-3329.

(9) Brigham, M. D.; et al. Mechanically Robust and Bioadhesive Collagen and Photocrosslinkable Hyaluronic Acid Semi-Interpenetrating Networks. Tissue Eng., Part A 2009, 15, 1645-1653.

(10) Collins, M. N.; Birkinshaw, C. Comparison of the effectiveness of four different crosslinking agents with hyaluronic acid hydrogel films for tissue-culture applications. J. Appl. Polym. Sci. 2007, 104, 3183-3191.

(11) Calderon, L.; et al. Type II collagen-hyaluronan hydrogel-a step towards a scaffold for intervertebral disc tissue engineering. Eur. Cells Mater. 2010, 20, 134-148.

(12) Lai, J.-Y. Biocompatibility of chemically cross-linked gelatin hydrogels for ophthalmic use. J. Mater. Sci.: Mater. Med. 2010, 21, 1899-1911.

(13) Jun, I.; et al. Preparation of Biomimetic Hydrogels with Controlled Cell Adhesive Properties and Topographical Features for the Study of Muscle Cell Adhesion and Proliferation. Macromol. Biosci. 2012, 12, 1502-1513.

(14) Moreira Teixeira, L. S.; Feijen, J.; van Blitterswijk, C. A.; Dijkstra, P. J.; Karperien, M. Enzyme-catalyzed crosslinkable hydrogels: Emerging strategies for tissue engineering. Biomaterials 2012, 33, $1281-1290$.

(15) Fan, Z.; Zhang, Y.; Fang, S.; Xu, C.; Li, X. Bienzymatically crosslinked gelatin/hyaluronic acid interpenetrating network hydrogels: preparation and characterization. RSC Adv. 2015, 5, 1929-1936.

(16) Chen, Y.-C.; Su, W.-Y.; Yang, S.-H.; Gefen, A.; Lin, F.-H. In situ forming hydrogels composed of oxidized high molecular weight hyaluronic acid and gelatin for nucleus pulposus regeneration. Acta Biomater. 2013, 9, 5181-5193.

(17) Camci-Unal, G.; Cuttica, D.; Annabi, N.; Demarchi, D.; Khademhosseini, A. Synthesis and characterization of hybrid hyaluronic acid-gelatin hydrogels. Biomacromolecules 2013, 14, 1085-1092.

(18) Vanderhooft, J. L.; Alcoutlabi, M.; Magda, J. J.; Prestwich, G. D. Rheological properties of cross-linked hyaluronan-gelatin hydrogels for tissue engineering. Macromol. Biosci. 2009, 9, 20-28.

(19) Levett, P. A.; et al. A biomimetic extracellular matrix for cartilage tissue engineering centered on photocurable gelatin, hyaluronic acid and chondroitin sulfate. Acta Biomater. 2014, 10, 214-223.

(20) Kim, I. L.; Mauck, R. L.; Burdick, J. A. Hydrogel design for cartilage tissue engineering: a case study with hyaluronic acid. Biomaterials 2011, 32, 8771-8782.

(21) Pfeifer, C. G.; et al. Higher ratios of hyaluronic acid enhance chondrogenic differentiation of human MSCs in a hyaluronic acidgelatin composite scaffold. Materials 2016, 9, 381. 
(22) Murphy, C. M.; Matsiko, A.; Haugh, M. G.; Gleeson, J. P.; O'Brien, F. J. Mesenchymal stem cell fate is regulated by the composition and mechanical properties of collagen-glycosaminoglycan scaffolds. J. Mech. Behav. Biomed. Mater. 2012, 11, 53-62.

(23) Burnsed, O. A.; et al. Hydrogels derived from cartilage matrices promote induction of human mesenchymal stem cell chondrogenic differentiation. Acta Biomater. 2016, 43, 139-149.

(24) Poveda-Reyes, S.; et al. Reinforcing an Injectable Gelatin Hydrogel with PLLA Microfibers: Two Routes for Short Fiber Production. Macromol. Mater. Eng. 2015, 300, 977.

(25) Poveda-Reyes, S.; et al. Gelatin-Hyaluronic Acid Hydrogels with Tuned Stiffness to Counterbalance Cellular Forces and Promote Cell Differentiation. Macromol. Biosci. 2016, 16, 1311.

(26) Dvořáková, J.; et al. Chondrogenic differentiation of mesenchymal stem cells in a hydrogel system based on an enzymatically crosslinked tyramine derivative of hyaluronan. J. Biomed. Mater. Res., Part A 2014, 102, 3523-3530.

(27) Lee, F.; Chung, J. E.; Kurisawa, M. An injectable enzymatically crosslinked hyaluronic acid-tyramine hydrogel system with independent tuning of mechanical strength and gelation rate. Soft Matter 2008, 4,880 .

(28) Jin, R; et al. Enzymatically-crosslinked injectable hydrogels based on biomimetic dextran-hyaluronic acid conjugates for cartilage tissue engineering. Biomaterials 2010, 31, 3103-3113.

(29) Sanmartín-Masiá, E.; Poveda-Reyes, S.; Ferrer, G. G. Extracellular matrix-inspired gelatin/hyaluronic acid injectable hydrogels. Int. J. Polym. Mater. Polym. Biomater. 2017, 66, 280-288.

(30) Meyvis, T. K. L.; et al. A comparison between the use of dynamic mechanical analysis and oscillatory shear rheometry for the characterisation of hydrogels. Int. J. Pharm. 2002, 244, 163-168.

(31) Kolesky, D. B.; et al. 3D bioprinting of vascularized, heterogeneous cell-laden tissue constructs. Adv. Mater. 2014, 26, $3124-3130$

(32) Moshayedi, P.; et al. Systematic optimization of an engineered hydrogel allows for selective control of human neural stem cell survival and differentiation after transplantation in the stroke brain. Biomaterials 2016, 105, 145-155.

(33) Wang, L.-S.; Du, C.; Chung, J. E.; Kurisawa, M. Enzymatically cross-linked gelatin-phenol hydrogels with a broader stiffness range for osteogenic differentiation of human mesenchymal stem cells. Acta Biomater. 2012, 8, 1826-1837.

(34) Segura, T.; et al. Crosslinked hyaluronic acid hydrogels: A strategy to functionalize and pattern. Biomaterials 2005, 26, 359-371.

(35) Levett, P. A.; et al. Chondrocyte redifferentiation and construct mechanical property development in single-component photocrosslinkable hydrogels. J. Biomed. Mater. Res., Part A 2014, 102, 25442553.

(36) Mueller, M. B.; et al. Hypertrophy in mesenchymal stem cell chondrogenesis: Effect of TGF- $\beta$ isoforms and chondrogenic conditioning. Cells Tissues Organs 2010, 192, 158-166.

(37) Dang, P. N.; Solorio, L. D.; Alsberg, E. Driving Cartilage Formation in High-Density Human Adipose-Derived Stem Cell Aggregate and Sheet Constructs Without Exogenous Growth Factor Delivery. Tissue Eng., Part A 2014, 20, 3163-3175.

(38) Rao, N.; et al. A co-culture device with a tunable stiffness to understand combinatorial cell-cell and cell-matrix interactions. Integr. Biol. 2013, 5, 1344-1354.

(39) Ansari, S.; et al. Muscle Tissue Engineering Using Gingival Mesenchymal Stem Cells Encapsulated in Alginate Hydrogels Containing Multiple Growth Factors. Ann. Biomed. Eng. 2016, 44, $1908-1920$.

(40) Ye, K.; Cao, L.; Li, S.; Yu, L.; Ding, J. Interplay of Matrix Stiffness and Cell-Cell Contact in Regulating Differentiation of Stem Cells. ACS Appl. Mater. Interfaces 2016, 8, 21903-21913.

(41) Zhang, K.; et al. Strategy for constructing vascularized adipose units in poly(L-glutamic acid) hydrogel porous scaffold through inducing in-situ formation of ASCs spheroids. Acta Biomater. 2017, 51, $246-257$.
(42) Evanko, S. P.; Angello, J. C.; Wight, T. N. Formation of hyaluronan- and versican-rich pericellular matrix is required for proliferation and migration of vascular smooth muscle cells. Arterioscler., Thromb., Vasc. Biol. 1999, 19, 1004-1013.

(43) Knudson, W.; Loeser, R. F. CD44 and integrin matrix receptors participate in cartilage homeostasis. Cell. Mol. Life Sci. 2002, 59, 3644.

(44) Choh, S.-Y.; Cross, D.; Wang, C. Facile Synthesis and Characterization of Disulfide-Cross-Linked Hyaluronic Acid Hydrogels for Protein Delivery and Cell Encapsulation. Biomacromolecules 2011, 12, 1126-1136. 